\title{
Expression and distribution of Toll like receptors in the brain during murine neurocysticercosis
}

\author{
Bibhuti B. Mishra ${ }^{1}$, Pramod K. Mishra ${ }^{2}$, and Judy M. Teale ${ }^{1,2,{ }^{*}}$ \\ 1 Department of Biology, The University of Texas at San Antonio, One UTSA Circle - San Antonio, \\ Texas 78249-1644. \\ 2 Department of Microbiology, The University of Texas Health Science Centre at San Antonio, San \\ Antonio, Texas 78229.
}

\begin{abstract}
In a mouse model of neurocysticercosis, the expression and distribution of Toll like receptors (TLRs) was investigated by using both gene array analyses and in situ immunofluoresence microscopy (IF). In the normal uninfected brain, mRNA of all the TLRs are constitutively expressed albeit TLR5, TLR7, TLR8 and TLR9 to a lesser extent. In these animals, however, expression of TLR1, TLR3, TLR4 and TLR9 proteins was not detected. In contrast, parasite infection increased both gene and protein level expression of all the TLRs several fold except TLR5 where only the mRNA was upregulated. Importantly, TLRs were differentially distributed among various central nervous system (CNS) cell types and infiltrating leukocytes. TLR2 was almost exclusively localized to nervous tissue cells, particularly astrocytes, while TLR1 and TLR9 proteins were essentially limited to infiltrating leukocytes. All other TLRs tested were detected in both CNS and immune cell types. Interestingly, ependymal cells and neurofilaments of the cerebellar white matter of infected mice exhibited a substantial upregulation of TLR7 and TLR8 protein respectively. These data provide a comprehensive analysis of TLR expression in the normal and parasite infected brain and suggest a role for TLRs in the interplay of immune cells and CNS cells during infection.
\end{abstract}

\section{Keywords}

Toll-like receptors; CNS; neurocysticercosis; parasite; helminth

\section{INTRODUCTION}

The CNS is regarded as an immunologically unique organ due to the absence of a classically defined lymphatic drainage system and the presence of the blood brain barrier (BBB) that restricts the flow of both cells and serum molecules into the CNS. During infection, however, cells of the CNS have the ability to produce inflammatory mediators such as chemokines, adhesion molecules and cytokines (Chavarria and Alcocer-Varela, 2004). These responses can lead to significant infiltration of various leukocytes eventuating in pathogen specific adaptive immune responses in the CNS (Ransohoff et al., 2003). The direct recognition of microbial molecules by nervous tissue cells and the subsequent innate immune response appear to be key elements in protecting the CNS. However, little is known about this process in a helminth parasitic infection of the CNS.

\footnotetext{
*Send correspondence and reprint requests to: Judy M. Teale, PhD, Department of Biology, The University of Texas at San Antonio, One UTSA Circle - San Antonio, TX 78249-1644; Tel: (210) 458-7024; Fax: (210) 458-7025; Email: judy.teale@utsa.edu. 
Recent studies have demonstrated the critical role played by the TLR family of proteins in host innate immunity (Hoebe et al., 2004). They are highly conserved proteins that specifically recognize distinct mutation resistant pathogen associated molecular patterns (PAMPs) (Takeda et al., 2003). Thirteen mammalian TLR paralogues have now been identified (10 in humans and 12 in mice) (Beutler, 2004). The TLR family receptors are type-I transmembrane proteins with extracellular leucine rich repeat domains and a cytoplasmic domain having significant homology to IL-1 receptor type-I called Toll/IL-1 receptor (TIR) domain (Bowie et al., 2004; Gay and Keith, 1991). Once engaged, signaling through TLRs starts from the TIR domain (Medzhitov, 2001) and involves one of the four known adaptor proteins: MyD88 (myeloid differentiation factor 88), MAL/TIRAP (MyD88-adaptor-like/TIR-associated proteins), TRIF (Toll-receptor-associated activator of interferons) and TRAM (Toll-receptor-associated molecule). The cascade of events that follows culminates in the production of immunoregulatory chemokines, cytokines, adhesion and co-stimulatory molecules (Dabbagh and Lewis, 2003; Takeda et al., 2003).

It has recently been shown that several nervous tissue cells upregulate particular TLRs as a result of infection, trauma, or autoimmune disease (Bsibsi et al., 2002; Glezer and Rivest, 2004; Sterka et al., 2006). Although, the exact functional significance of TLRs in the context of CNS diseases is largely unknown, a recent study using TLR2 -/- mice in an S. aureus induced experimental brain abscess model indicated a reduction of proinflammatory mediators, but the rate of mortality and abscess severity were found to be comparable (Kielian et al., 2005b). In contrast in experimental pneumococcal meningitis, mice deficient in TLR2 were highly susceptible to Streptococcus pneumoniae meningitis because of reduced bacterial clearance, but host inflammatory mediators appeared largely unaffected (Echchannaoui et al., 2002; Koedel et al., 2003). In mice lacking MyD88, the adaptor protein involved in downstream signaling of most of the TLRs (Takeda et al., 2003), a reduced turnover of immune mediators and inflammation was detected in the CNS (Koedel et al., 2004), perhaps supporting involvement of additional TLRs. Thus, a systematic study of infection induced expression of a number of TLRs is warranted, particularly in the control of a parasitic infection that has been understudied.

In an experimental murine model for NCC (Cardona et al., 2003), gene specific arrays were used to detect TLRs 1-9 at the RNA level for both infected and mock infected mice. In situ IF microscopy was performed to define the differential expression of TLRs on particular cell types in infected and uninfected brains using antibodies specific for each of the TLRs in combination with antibodies for distinct cell surface markers to either CNS cells or immune cells. Our findings define the TLR expression profile of cell types in normal and parasitic infected brain and implicate TLR-mediated immune surveillance as an important component of immune defense in the CNS.

\section{MATERIALS AND METHODS}

\section{Mice}

Female Balb/c mice were used in this study. Mice were purchased from the National Cancer Institute animal program (Bethesda, MD). Experiments were conducted under the guidelines of the IACUC, UTHSCSA, University of Texas System, the US Department of Agriculture, and the National Institutes of Health.

\section{Antibodies}

Anti-mouse TLRs 2, 3, 4, 6 and 9 antibodies were purchased from Imgenex (San Diego, CA). Antibodies against human TLRs 1, 7 and 8 along with the corresponding synthetic peptides used as antigens were purchased from Zymed (San Francisco, CA). These antibodies were 
developed against KLH-conjugated synthetic peptides of respective TLRs from human origin. Due to sequence homology it was expected to react with corresponding mouse counterparts. This was found to be correct and specificity was confirmed by the ability of the relevant synthetic peptides to block antibody binding. Anti-mouse CD11b (myeloid cells) either biotinylated or PE conjugated, biotinylated anti-mouse CD11c (dendritic cells), PE conjugated anti-mouse CD19 (B-Cells), and PE conjugated anti-TCR of $\alpha \beta$ and $\gamma \delta$ T cells were purchased from Pharmingen (San Diego, CA). Biotinylated anti-mouse Neun (neuronal nuclear protein) was purchased from US biological (Swampscott, MA). Anti-mouse neurofilament (Santa Cruz Biotech, CA) and anti-mouse GFAP (Pharmingen) was later conjugated to Alexa Fluor 488 (Molecular Probes) according to manufacturers' instructions. For indirect immunofluoresence, the primary antibody binding of rabbit anti-TLRs 1, 2, 5, 6, 7, 8 and 9, and rat anti mouse TLRs 3 and 4 were detected with the corresponding Cy2 conjugated (green) or Cy3 conjugated (red) goat anti-rabbit and goat anti-rat secondary antibodies respectively (Jackson laboratory). Biotinylated primary antibodies were detected using Alexa Fluor 488 labeled streptavidin (Molecular Probes).

\section{Murine model of neurocysticercosis}

A well characterized mouse model of neurocysticercosis was used in this study (Cardona et al., 1999; Cardona and Teale, 2002). Mice were infected with the larval stage of Mesocestoides corti parasites. Briefly, parasites were maintained by serial intraperitoneal (i.p.) inoculations of 6-8 wk old female BALB/c mice. Murine neurocysticercosis was induced by intracranial injection of $40 \mu \mathrm{l}$ of HBSS containing about 40 parasites into 3-5 week old mice under short term anesthesia.

\section{RNA isolation and gene array analysis}

To determine the CNS gene expression of TLRs in murine neurocysticercosis, brains were removed from infected mice at 1,2, 4, 6 and 10wk post infection (p.i.) in addition to vehicle control animals ( $\mathrm{n}=2$ in each group). Animals were perfused prior to sacrifice to avoid RNA contamination from blood cells. Briefly, mice were anaesthetized with $100 \mu \mathrm{l}$ of mouse cocktail containing $100 \mathrm{mg} / \mathrm{ml}$ of ketamine and $20 \mathrm{mg} / \mathrm{ml}$ of rompum (Laboratory animal resource, UTHSCSA, TX) and perfused with $10 \mathrm{ml}$ of PBS, pH 7.4 through the left ventricle. The brain was immediately removed after perfusion and total RNA was extracted using Trizol reagent (Invitrogen) according to manufacturers' instructions. Levels of TLR specific RNA in these samples were measured by using the GEarray $Q$ series NFkB pathway-specific gene expression array from SuperArray according to the manufacturers' instructions. Four micrograms of total RNA from each sample was reverse transcribed into cDNA in the presence of $\alpha-{ }^{33} \mathrm{P}-\mathrm{dCTP}$ using the templates and reverse transcriptase supplied in the kits. The resulting cDNA probes were hybridized to TLR gene specific cDNA fragments that were spotted as a tetra spot on the GEArray membranes. Each of the blots had several spots either blank or with cDNA fragments of PUC18 plasmid to judge the specificity of the hybridization. The radioactive signals corresponding to the bound probes were measured using the Typhoon ${ }^{\mathrm{TM}} 9400$ phosphor imaging system (Molecular Dynamics, Sunnyvale, CA). The numerical value corresponding to the TLR gene band intensities was recorded and corrected for the background level of each blot using Image Quant software (Molecular Dynamics, Sunnyvale, CA). The raw data values were further analyzed using the GEArray software. The averaged intensity of each TLR gene in arbitrary units was normalized to the averaged values recorded for two house keeping genes, cyclophilin A and rpl13a which were present as internal controls in each blot. The normalized value represents mRNA expression for each TLR gene. 


\section{Immunofluorescence}

To detect tissue expression and distribution of TLRs in the CNS of parasite infected mice, brains were analyzed from mice after various times post infection (3d, 1wk, 11d, 2wk, 3wk, $4 \mathrm{wk}, 5 \mathrm{wk}, 7 \mathrm{wk}$ and $10 \mathrm{wk}$ ) in addition to brains from mock infected control animals. The brain was immediately removed after perfusion (as described before), embedded in optimal cutting temperature (OCT) medium, and snap frozen as described before (Cardona et al., 2003). Serial horizontal cryosections of $10 \mu \mathrm{m}$ in thickness were placed on silane prep slides (Sigma Biosciences, St. Louis, Mo). One in every four slides was fixed in formalin for $12 \mathrm{~min}$ at room temperature and stained with hematoxylin and eosin. The remainder of the slides was air dried overnight and fixed in fresh acetone for $20 \mathrm{~s}$ at room temperature. Acetone-fixed sections were wrapped in aluminum foil and stored at $-80^{\circ} \mathrm{C}$ or processed immediately for immunofluorescence.

Immunofluorescence analysis was performed to determine the presence of individual TLRs in brain sections. All the steps were carried out at room temperature. Sections were incubated with TLR specific primary antibodies in PBS buffer with 3\% host serum to prevent non-specific binding. After $1 \mathrm{hr}$, sections were washed seven times at 3 min each and incubated with appropriate secondary antibodies for $30 \mathrm{~min}$. Sections were then washed seven times $3 \mathrm{~min}$ each in $50 \mathrm{mM}$ Tris-HCl, pH 7.6 with $0.1 \%$ Tween-20. For two or three color staining the above mentioned procedures were sequentially repeated for each additional staining. The sections were mounted using Fluro save reagent (Calbiochem, La Jolla, CA) containing 0.3 $\mu \mathrm{M}$ 4', 6'-diamidino-2-phenylidole (DAPI)-diacetate (Molecular Probes). Additional control staining was performed to rule out any nonspecific staining. In each case, sections were blocked with saturating concentrations of appropriate isotype control antibodies and/or host serum antibodies to eliminate false positives due to FcR mediated nonspecific binding. In addition, staining in absence of the primary antibodies was performed as negative controls.

In this study anti-CD11b staining was used to identify both microglia of the nervous tissue and infiltrating leukocytes. A potential problem is anti-CD11b stains a number of cell types that include leukocytes such as macrophages, neutrophils, plasmacytoid dendritic cells (pDCs) and microglia. In our previous studies involving murine NCC (Alvarez and Teale, 2006; Cardona et al., 1999) we used a number of cell surface markers e.g. CD11b, F4/80, anti-mouse monocytes/macrophages (MOMA-2), IsoB4, neutrophil elastase, Gr-1, DEC205, and CD11c. Using these markers together with morphology and cellular distribution, CD11b+ cells are typically observed in relatively large numbers as clusters of cells in meninges, ventricles, and parenchyma proximal to parasites and are presumed to be infiltrating macrophages. However, as activated $\mathrm{CD} 11 \mathrm{~b}+$ microglia can change to an amoeboid shape and migrate, we refer to these cells as infiltrating CD11b+ myeloid cells. In contrast, CD11b+ cells which exhibit characteristic ramified microglia-like morphology and are evenly distributed in the parenchyma are presumed to be microglia.

\section{RESULTS}

\section{mRNA Expression of TLRs in normal and infected brain tissue}

The expression and distribution of TLRs following in vivo helminth infection has not been reported. As a first step we performed GEArray analysis to observe the expression profiles of genes for TLR 1-9 in both uninfected and parasite infected brain. In all the blots the absence of false positive signals on the blank and PUC18 plasmid DNA coated spots confirmed the specificity of the signal. In normal and mock infected brain constitutive mRNA expression could be detected for all of the TLRs (Fig. 1A) with a relatively higher level of gene expression of TLRs 2, 3 and 4 and a lower level of expression for TLRs 1, 5, 7, 8 and 9. Upon infection, expression of TLR genes 1-9 was upregulated substantially (Fig. 1B). The extent of increased 
gene expression was greatest at $1-2 \mathrm{wk}$ p.i. than at later stages ( $4 \mathrm{wk}, 6 \mathrm{wk}$ and $10 \mathrm{wk}$ ). These data were reproducible in two independent sets of experiments ( $\mathrm{n}=2$ at each time point). These results indicate the presence of mRNA for all of the TLRs tested in normal mouse brain and a substantial increase as a result of CNS infection. Furthermore, TLR mRNA expression detected by membrane microarrays was validated by Real Time PCR analysis for TLRs 1, 2, 6 and 7 in infected (and uninfected) mice using SYBR green as detection dye. In NCC mice at $1 \mathrm{wk}$ and 2 wk p.i., these TLR genes were upregulated by 4 to 8 fold (data not shown). This further confirms the increase in mRNA expression of multiple TLRs during NCC.

\section{Expression and distribution of TLRs 1-9 proteins among nervous tissue and infiltrating immune cell types}

The distribution of individual TLR proteins was examined by in situ IF microscopy in brain tissues from mock infected control mice and NCC mice. Brains were analyzed at 3d, 1wk, 11d, $2 w k, 3 w k, 4 w k, 5 w k, 7 w k$ and $10 w k$ p.i. At least two mice were analyzed per time point with reproducible results.

On the basis of similarity in amino acid composition and microbial ligand binding pattern, TLRs can be divided into five subfamilies viz. TLR2 subfamily- TLR1, 2, and 6; TLR9 subfamily- TLR7, 8, and 9; and TLR3, TLR4 and TLR5 subfamilies each consisting of a single member (Takeda et al., 2003). Representative fluorescent images are shown for each TLR together with selected cell type specific markers (Fig. 2-5). However, a complete summary of the distribution profile of individual TLRs on distinct cell types is provided in Table 1.

TLR2 family-TLR1 was not detected in normal mouse brain at the protein level by IF staining (Fig. 2A1). A few TLR1 positive infiltrating cells were observed at $3 \mathrm{~d}$ p.i. in meninges, but a striking increase in the number of TLR1 positive infiltrating cells was found at 1wk p.i. in meninges (Fig. 2A2) and ventricles. As the length of time p.i. increased, there was a progressive decline in the number of TLR1 positive leukocytes and their location. By 4wk p.i., TLR1 positive infiltrating cells were more evident in parenchyma than in ventricles and meninges. Double immunofluorescence was performed with anti-TLR1 and antibodies to cell surface markers specific for macrophages/microglia cells (CD11b), dendritic cells (CD11c), B-cells (CD19), $\gamma \delta$ T (TCR $\delta$ chain) and $\alpha \beta$ T cells (TCR $\beta$ chain). Colocalization of TLR1 was found only with CD11b and $\gamma \delta$ T cells (Fig. 2A3, 4, yellow color in merged image). Except for a few microglia that were positive for TLR 1 in the cerebellum of normal mouse brain and a few neurons in cortical regions of $7 \mathrm{wk}$ and 10wk p.i. mice brain, TLR1 staining was almost exclusively detected in infiltrating cells (Table 1).

TLR2 staining was evident in brain tissue cells of both uninfected and infected mice (Fig. 2B1-4). Brain tissue cells were defined by their characteristic morphology together with cell markers: microglia (CD11b), astrocytes (GFAP), and neurons (NeuN). TLR2 expression was detected at a low, apparently basal level in microglia, neurons, ependymal cells and astrocytes in normal and mock infected control brains (Fig 2B1). In these uninfected animals, TLR2 expressing astrocytes were detected mostly in the periventricular and leptomeningeal (LM) areas. TLR2 positive microglia and neurons were detected mostly in the parenchyma. After 3 days p.i., an increased expression of TLR2 was evident in astrocytes and on their processes present in periventricular and LM areas of the brain. This upregulation was more dramatic after 1wk p.i (Fig. 2B2). Although an increase in TLR2 expression was found in microglia (Fig. 2B4, yellow/orange color in merged image), neurons, and ependymal cells, the most striking increase in expression was observed with astrocytes and their processes (Fig. 2B3). TLR2 positive astrocytes could be detected in every area of the brain examined and multiple sections of brains were analyzed. In the later phase of infection, these induced effects were even more pronounced and appeared to reach a maximal expression level at 4wk p.i. as measured by both 
intensity of staining and number of positive cells. After 4 wk p.i., TLR2+ cells staining progressively decreased. Very few infiltrating cells exhibited expression of TLR2 irrespective of the length of time post infection (Table 1).

TLR6 staining was detected on few nervous tissue cells in normal or mock infected mouse brain (Fig. 2C1). In NCC mouse brain TLR6 was expressed in both nervous tissue and infiltrating immune cells (Fig. 2C2-4, Table 1). TLR6 expression in the nervous tissue was similar to TLR2 expression with respect to both cell types and their location (Fig. 2C2), although the number of positive cells appeared fewer than TLR2 positive cells. TLR6 staining was most striking in astrocytes (Fig. 2C3). Staining was also evident on infiltrating CD11b+ myeloid cells (Fig. 2C4) and $\gamma \delta$ T cells but not on $\alpha \beta$ T cells or B cells (Table 1). The maximal number of cells expressing TLR6 was observed at $2 \mathrm{wk}$ p.i.; this is in contrast to the maximal staining observed for TLR1 in 1wk and TLR2 in 4wk NCC brain respectively.

TLR3-The basal brain level of TLR3 mRNA was relatively high in uninfected control animals but TLR3 protein was not detected (Fig. 3A1). Upon infection TLR3 appeared to be upregulated predominantly on astrocytes (Fig. 3A2) which was confirmed by colocalization with GFAP (Fig. 3A3, yellow/orange color). Other CNS cell types were negative (Table 1). Among infiltrating cells, both CD11b+ myeloid cells (Fig. 3A4; Table 1), and $\gamma \delta$ T cells were found to express TLR3 (Table 1). Interestingly the kinetics of expression of TLR3 was different in astrocytes and infiltrating $\gamma \delta \mathrm{T}$ and CD11b positive cells. The number of astrocytes expressing TLR3 was first detected by 1 wk p.i., peaked by 4 wk p.i. and remained high throughout $7 w k s$ of infection. TLR3 expressing $\gamma \delta \mathrm{T}$ and infiltrating CD11b+ myeloid cells were not detected until 2 wks p.i. even though both $\gamma \delta \mathrm{T}$ cells and CD11b positive leukocytes infiltrate the CNS as early as 1-3 days p.i. as previously reported (13).

TLR4-TLR4 expression at the protein level was not detected in brain from normal or mock infected control mice (Fig. 3B1). Upon infection, TLR4 was evident on microglia and neurons (Fig. 3B2, B3; Table 1). The number of TLR4 positive neurons gradually increased during infection and at the later stages of infection (4-10 wks) appeared highly activated as indicated by the enlarged cell bodies (Fig. 3B3). The presence of TLR4 was undetectable in astrocytes and oligodendrocytes. In terms of infiltrating leukocytes, TLR4 expression appeared to be restricted to CD11b+ myeloid cells (Fig. 3B4; Table 1), and $\gamma \delta$ T cells (Table 1).

TLR5-TLR5 recognizes flagellin, a bacterial protein component of gram-negative bacteria (Hayashi et al., 2001). None of our experimental brain samples from either control or NCC infected brains exhibited expression of TLR5 protein (Table 1). This is conspicuous considering the gene expression level of TLR5 was comparable to that of TLR1, 6, 7, 8 and 9 in both mock infected control and infected brain (Fig. 1). The TLR5 specific antibody used here worked well on frozen lung sections from mice infected with Francisella tularensis (data not shown).

TLR9 family-TLR7, 8 and 9 positive cells were few in number or absent in normal or mock infected mice (Fig. 4A1, B1, \& C1). TLR7 staining was exclusively localized to ependymal cells lining the ventricles (Fig. 4A1, arrows). TLR8 positive cells in these animals were few in number and mostly confined to cells in the white matter of periventricular, subcortical and cerebellar regions of the brain (Fig. 4B1). These TLR8 positive cells looked morphologically similar to astrocytes or oligodendrocytes. TLR9 protein expression was barely detectable in these animals (Fig. 4C1).

Next the expression profile of these TLRs during NCC was determined (Fig. 4, Table 1). By 1 wk p.i., expression of TLRs 7, 8 and 9 were substantially upregulated. TLR7 expression was particularly interesting. At $1 \mathrm{wk}$ post infection almost all the ependymal cells were abundantly 
positive for TLR7 (Fig. 4A2; arrow). In addition, substantial numbers of cerebellar granular neurons (Fig. 4A2; stars), and vascular endothelial cells were found to be positive for TLR7 (data not shown). TLR7 staining was also evident in astrocytes (Fig. 4A3) and some microglia present in the cerebellar and periventricular white matter (Table 1). Among the infiltrating cells, the presence of TLR7 was observed on a substantial number of CD11b+ myeloid cells (Fig. 4A4) and $\gamma \delta \mathrm{T}$ cell positive cells between $1 \mathrm{wk}$ to $5 \mathrm{wk}$ p.i. (Table 1 ).

Parasite infection of brain resulted in substantial upregulation of protein expression for both TLR8 and TLR9 but predominantly in infiltrating cells present in ventricles and meninges (Fig. 4B2 \& C2). This was evident from $1 \mathrm{wk}$ to $5 \mathrm{wk}$ p.i. At later stages of infection ( $7 \mathrm{wk}$ and $10 \mathrm{wk}$ p.i.), however, it was hard to find any infiltrating cells expressing TLR8 and 9 in mouse brain. The majority of infiltrating cells during the $1 \mathrm{wk}$ to $5 \mathrm{wk}$ post infection times that stained for both TLR 8 and 9 were CD11b+ myeloid cells and $\gamma \delta$ T cells (Fig. 4B4 \& C3, C4; Table 1). In addition few B-cells and $\alpha \beta T$ cells were found to express TLR8 between $3 \mathrm{wk}$ and $5 \mathrm{wk}$ p.i. (Table 1), although the number of such cells was low. Interestingly axons originating from the granular neurons and projecting through the cerebellar white matter were distinctly positive for TLR8 in all NCC brain tissue tested after 1wk of infection (Fig. 4B3). Several of these TLR8 positive neurofilaments appeared to be in close contact with CD11c positive dendritic/ microglia cells (data not shown).

\section{DISCUSSION}

Previous studies have shown that pathogens and their derived antigens can induce a robust immune response that causes persistent inflammatory responses in the CNS. Frequently in CNS diseases, sustained inflammation is the cause of tissue damage and associated pathology (Chavarria and Alcocer-Varela, 2004). The neuroinflammatory responses involve infiltrating peripheral cells of the immune system and nervous tissue cells, all participating in a complex orchestration of coagulation factors, complement, proteases and among others cytokines predominantly with a Th1 phenotype (McGeer and McGeer, 2001). Although TLR signaling results in inflammatory/Th1 responses in the peripheral organs (Aliprantis et al., 1999; Brightbill et al., 1999; Dabbagh and Lewis, 2003; Pulendran, 2004) their role in the CNS is largely unknown. One of the major limitations has been a lack of knowledge about their distribution among CNS cell types. As in several other CNS diseases, murine NCC caused by the helminth $M$. corti is typically associated with infiltration of large numbers of immune cells and a predominant TH1 inflammatory response (Cardona et al., 1999; Cardona and Teale, 2002). The present study used a murine NCC model to determine the cellular distribution of TLR proteins in brain under normal as well as after infection. This is the first systematic report characterizing TLR protein expression in situ in different CNS cells from normal and infected mice as well as leukocytes infiltrating the brain upon parasitic infection. Importantly infection by $M$. corti resulted in the upregulation of all of the TLRs tested except TLR5 which is likely reflective of the complexity of helminth parasites.

Our findings indicate that mRNA for all the TLRs tested was detected in normal uninfected mouse brain but at relatively low levels. Other studies have reported that astrocytes express low-level constitutive mRNA expression of TLRs 2, 4, 5 and 9, and activation with specific TLR ligands resulted in further elevation in their expression (Bowman et al., 2003). A subsequent study has shown that in addition to TLRs 2, 4, 5, and 6, resting astrocytes also express moderate levels of TLRs 1 and 6 , and none or very low level of TLRs 7 and 8 (Carpentier et al., 2005). Two other previous studies also showed detectable levels of gene expression of TLRs 1-10 in human brain (Bsibsi et al., 2002), and TLRs 1-9 in isolated murine microglia (Olson et al., 2004). However, despite the presence of TLR specific mRNA, our results indicate that not all of these TLRs appear to be expressed as proteins, suggesting that several of them are post transcriptionally regulated. Interestingly, in uninfected mice the 
periventricular and meningeal areas, CNS tissues that are likely to be exposed early in infection to pathogens or their derived molecules (Ransohoff et al., 2003) are detected positive for TLR2 (Laflamme and Rivest, 2001; Laflamme et al., 2001) 6, 7 and 8. Among all the TLRs, TLR2 expression in the uninfected brain was most notable in terms of the number of TLR2 positive nervous tissue cells and the intensity of staining. The data are consistent with the current belief that TLR2 is the most prominent among the TLRs. TLR2 recognizes a broad spectrum of microbial components that include molecules from organisms as diverse as bacteria, mycobacterium, fungi, yeast, protozoan parasites and spirochetes (Michelsen et al., 2001; Nishiguchi et al., 2001; Takeuchi and Akira, 2002; Zanin-Zhorov et al., 2003). This diversity of TLR2 ligands may account for its prominent expression during homeostasis so that a rapid response can be mounted once infection occurs.

In the NCC model, acute infection with $M$. corti causes substantial upregulation of mRNA for all of the TLRs tested. However, in contrast to the mock control brain, protein expression of all of the TLRs except TLR5 was detected. Importantly, they were differentially expressed on brain tissue cells and infiltrating leukocytes. TLRs 1 and 9 were found almost exclusively on infiltrating leukocytes, TLR2 on brain tissue cells, and the remainder on both cell types. This raises several questions regarding individual TLR mediated effector functions, potential cross talk between nervous tissue cells and infiltrating leukocytes, and the extent to which each of the TLR mediated signaling events is associated with immune function. Recent studies indicate that nervous tissue cells through TLR signaling are associated with inflammatory responses by mediating induction of chemokines, cytokines, and costimulatory molecules (Carpentier et al., 2005; Esen et al., 2004; Lee et al., 2004; Olson et al., 2004; Olson and Miller, 2004). With $M$. corti infection, the astrocyte marker GFAP colocalized with TLR2, 3, 6, and 7. The expression of TLRs on astrocytic foot processes that terminate at blood vessels might provide necessary signals for subsequent infiltration of immune cells to the CNS (Abbott, 2002).

Positive staining for several TLRs was also present on CD11b positive parenchymal microglia. Activation of microglia plays an important role in leukocyte trafficking to this area through their effects on the blood brain barrier (Nguyen et al., 2002), and TLR signaling may lead to production of host inflammatory mediators which in turn may play a significant role in this process (Kielian et al., 2005a). Induction of TLRs in the infected/diseased brain for extended periods is likely integral to persistent inflammation and its associated pathology (Koedel et al., 2004; Nguyen et al., 2002; Turrin and Rivest, 2004).

It is interesting that the only TLR protein not induced during this infection is TLR5 for which the known ligand is flagellum (Takeda et al., 2003). Relative to viruses, bacteria, and intracellular parasites, only a limited amount of work has been done on the role of TLRs in response to extracellular pathogens, including helminths. Recent studies have reported two egg derived components from the parasite Schistosoma mansoni, the glycolipid lysophosphatidylserine and the carbohydrate determinant acto-N-fucopentaose III activate TLR2 and TLR4, respectively (Thomas et al., 2003; van der Kleij et al., 2002). Also the egg stage contains dsRNA that activate TLR3 in myeloid dendritic cells (Aksoy et al., 2005). In addition, phosphorylcholine containing secretory components of filarial nematode activates TLR4 (Goodridge et al., 2005) and carbohydrates of Taenia crassiceps carbohydrates has been postulated to stimulate IL6 expression through TLRs (Dissanayake et al., 2004). M. corti being a multicellular helminth parasite with a diversity of antigens likely contains unique motifs that recognize TLR receptors and directly elicits inflammatory responses in the brain. Other studies from our laboratory currently being prepared for publication indicate that murine NCC is associated with release of parasitic glycoproteins and glycolipids throughout the active infection period. Some of these molecules likely act as specific PAMPs for particular TLRs that have not yet been described. In this light it is interesting to note that TLR1 and TLR6 were detected in infiltrating CD11b+ myeloid cells, while TLR2 is negligible. 
One aspect of TLR2 ligand recognition in the peripheral immune system normally involves TLR2 dimerization with TLR1 or 6 (Takeda et al., 2003). The similarity in the expression pattern of both TLR2 and TLR6 but not TLR1 among nervous tissue cell types suggests the functional association of TLR2 and TLR6 on the nervous tissue cells analyzed. However, the presence of TLR1 and 6 on infiltrating cells when TLR2 was undetectable is puzzling. One possibility is that helminth parasites are notorious for their ability to immunomodulate the immune response and downregulation of TLR2 may be one such mechanism. Another possibility is that some of the M. corti secreted antigens induce TLR1 and TLR6 homodimers or a TLR1 and 6 heterodimer, a hypothesis currently being tested in our laboratory using parasite specific antigens.

It is also likely that infection induced cytokines indirectly induce the expression of particular TLR proteins (Matsuguchi et al., 2000). As discussed in the text, upregulation of the majority of TLRs analyzed in infected mice occurred prior to upregulation in infiltrating leukocytes (e.g. TLR2, 3, 4, and 6). This is of interest in that the infiltration of leukocytes occurs as early as 1-3 days post infection. Moreover, our previous studies have shown that IL6 and vascular endothelial growth factor (VEGF) are expressed in the first several days of infection (Alvarez and Teale, 2006). Thus, cytokines produced by TLR activated nervous tissue cells may be involved in upregulation of TLR proteins on infiltrating leukocytes. Presumably, leukocyte derived cytokines could in turn affect the regulation of TLR function in CNS cells. In the light, it is interesting that at 7-10wks p.i. the majority of TLR proteins are still upregulated on CNS cells but not infiltrating cells. Perhaps this reflects a neuroprotective role of TLRs. Regardless; the in vivo data suggest a complex interplay between nervous tissue cells and infiltrating leukocytes. In vitro studies are underway to define such interactions.

A critical question is whether TLRs have other functions besides inflammation that may be important in diseases of the CNS. In this light, constitutive expression and infection-induced upregulation of particular TLR proteins in distinct nervous tissue cell types is intriguing. Upregulation of TLR7 proteins in ependymal cells is especially interesting. Ependymal cells are specialized neural stem cell types in adult CNS (Rietze et al., 2001), having intrinsic ability to rapidly proliferate and produce both neurons and astrocytes during injury (Johansson et al., 1999). In addition, TLRs are presumed to participate in innate immune response mediated neuroprotection (Nguyen et al., 2002). Colocalization of TLR8 proteins with neurofilaments projecting through the cerebellar white matter in NCC brain is also interesting (Fig 4B3). Presently, the function of TLR8 is not known in mice (Heil et al., 2004). In humans, TLR8 was previously reported only in intracellular compartments, and it was speculated that its natural ligands were located in acidic compartments such as phagolysosomes (Ulevitch, 2004). TLR8 in the CNS cerebellar region is likely involved in another level of ligand recognition as the white matter is devoid of neuronal cytoplasm. This may suggest the presence of TLR8 on the surface of axons. Interestingly, these TLR8 positive neurofilaments appear in close association with CD11c positive dendritic/microglia cells. Defining the distinct role of TLRs in brain cells and their potential interaction with the immune system will be critical for understanding CNS disease pathology.

The differential expression of particular TLRs on both nervous tissue cells and infiltrating leukocytes suggests an important but complex role for TLRs in the CNS immune response to M. corti infection. An enhanced understanding of TLR function in specific CNS cell types and their mechanism of ligand recognition should provide insight into their role in initiation of immune reactions in CNS infection and the deleterious sequelae associated with chronic infections, particularly parasitic infection. 


\section{Acknowledgements}

We gratefully acknowledge the technical support of Ms. Xiaojing Xu and the secretarial support of Ms. Marguerite Starr.

\section{References}

Abbott NJ. Astrocyte-endothelial interactions and blood-brain barrier permeability. J Anat 2002;200:629-638. [PubMed: 12162730]

Aksoy E, Zouain CS, Vanhoutte F, Fontaine J, Pavelka N, Thieblemont N, Willems F, RicciardiCastagnoli P, Goldman M, Capron M, Ryffel B, Trottein F. Double-stranded RNAs from the helminth parasite Schistosoma activate TLR3 in dendritic cells. J Biol Chem 2005;280:277-283. [PubMed: 15519998]

Aliprantis AO, Yang RB, Mark MR, Suggett S, Devaux B, Radolf JD, Klimpel GR, Godowski P, Zychlinsky A. Cell activation and apoptosis by bacterial lipoproteins through toll-like receptor-2. Science 1999;285:736-739. [PubMed: 10426996]

Alvarez JI, Teale JM. Breakdown of the blood brain barrier and blood-cerebrospinal fluid barrier is associated with differential leukocyte migration in distinct compartments of the CNS during the course of murine NCC. J Neuroimmunol 2006;173:45-55. [PubMed: 16406118]

Beutler B. Innate immunity: an overview. Mol Immunol 2004;40:845-859. [PubMed: 14698223]

Bottcher T, von Mering M, Ebert S, Meyding-Lamade U, Kuhnt U, Gerber J, Nau R. Differential regulation of Toll-like receptor mRNAs in experimental murine central nervous system infections. Neurosci Lett 2003;344:17-20. [PubMed: 12781911]

Bowie AG, Zhan J, Marshall WL. Viral appropriation of apoptotic and NF-kappaB signaling pathways. J Cell Biochem 2004;91:1099-1108. [PubMed: 15048867]

Bowman CC, Rasley A, Tranguch SL, Marriott I. Cultured astrocytes express toll-like receptors for bacterial products. Glia 2003;43:281-291. [PubMed: 12898707]

Brightbill HD, Libraty DH, Krutzik SR, Yang RB, Belisle JT, Bleharski JR, Maitland M, Norgard MV, Plevy SE, Smale ST, Brennan PJ, Bloom BR, Godowski PJ, Modlin RL. Host defense mechanisms triggered by microbial lipoproteins through toll-like receptors. Science 1999;285:732-736. [PubMed: 10426995]

Bsibsi M, Ravid R, Gveric D, van Noort JM. Broad expression of Toll-like receptors in the human central nervous system. J Neuropathol Exp Neurol 2002;61:1013-1021. [PubMed: 12430718]

Cardona AE, Gonzalez PA, Teale JM. CC chemokines mediate leukocyte trafficking into the central nervous system during murine neurocysticercosis: role of gamma delta $\mathrm{T}$ cells in amplification of the host immune response. Infect Immun 2003;71:2634-2642. [PubMed: 12704138]

Cardona AE, Restrepo BI, Jaramillo JM, Teale JM. Development of an animal model for neurocysticercosis: immune response in the central nervous system is characterized by a predominance of gamma delta T cells. J Immunol 1999;162:995-1002. [PubMed: 9916725]

Cardona AE, Teale JM. Gamma/delta T cell-deficient mice exhibit reduced disease severity and decreased inflammatory response in the brain in murine neurocysticercosis. J Immunol 2002;169:3163-3171. [PubMed: 12218134]

Carpentier PA, Begolka WS, Olson JK, Elhofy A, Karpus WJ, Miller SD. Differential activation of astrocytes by innate and adaptive immune stimuli. Glia 2005;49:360-374. [PubMed: 15538753]

Chavarria A, Alcocer-Varela J. Is damage in central nervous system due to inflammation? Autoimmun Rev 2004;3:251-260. [PubMed: 15246020]

Dabbagh K, Lewis DB. Toll-like receptors and T-helper-1/T-helper-2 responses. Curr Opin Infect Dis 2003;16:199-204. [PubMed: 12821808]

Dissanayake S, Amith RS, Shahin A. Taenia crassiceps carbohydrates stimulate IL-6 expression in naive murine macrophages via Toll-like receptors (TLRs). Mol Immunol 2004;41:391-398. [PubMed: 15163536]

Echchannaoui H, Frei K, Schnell C, Leib SL, Zimmerli W, Landmann R. Toll-like receptor 2-deficient mice are highly susceptible to Streptococcus pneumoniae meningitis because of reduced bacterial clearing and enhanced inflammation. J Infect Dis 2002;186:798-806. [PubMed: 12198614] 
Esen N, Tanga FY, DeLeo JA, Kielian T. Toll-like receptor 2 (TLR2) mediates astrocyte activation in response to the Gram-positive bacterium Staphylococcus aureus. J Neurochem 2004;88:746-758. [PubMed: 14720224]

Gay NJ, Keith FJ. Drosophila Toll and IL-1 receptor. Nature 1991;351:355-356. [PubMed: 1851964]

Glezer I, Rivest S. Glucocorticoids: protectors of the brain during innate immune responses. Neuroscientist 2004;10:538-552. [PubMed: 15534039]

Goodridge HS, Marshall FA, Else KJ, Houston KM, Egan C, Al-Riyami L, Liew FY, Harnett W, Harnett MM. Immunomodulation via novel use of TLR4 by the filarial nematode phosphorylcholinecontaining secreted product, ES-62. J Immunol 2005;174:284-293. [PubMed: 15611251]

Hayashi F, Smith KD, Ozinsky A, Hawn TR, Yi EC, Goodlett DR, Eng JK, Akira S, Underhill DM, Aderem A. The innate immune response to bacterial flagellin is mediated by Toll-like receptor 5 . Nature 2001;410:1099-1103. [PubMed: 11323673]

Heil F, Hemmi H, Hochrein H, Ampenberger F, Kirschning C, Akira S, Lipford G, Wagner H, Bauer S. Species-specific recognition of single-stranded RNA via toll-like receptor 7 and 8 . Science 2004;303:1526-1529. [PubMed: 14976262]

Hoebe K, Janssen E, Beutler B. The interface between innate and adaptive immunity. Nat Immunol 2004;5:971-974. [PubMed: 15454919]

Johansson CB, Momma S, Clarke DL, Risling M, Lendahl U, Frisen J. Identification of a neural stem cell in the adult mammalian central nervous system. Cell 1999;96:25-34. [PubMed: 9989494]

Kielian T, Esen N, Bearden ED. Toll-like receptor 2 (TLR2) is pivotal for recognition of S. aureus peptidoglycan but not intact bacteria by microglia. Glia 2005a;49:567-576. [PubMed: 15593098]

Kielian T, Haney A, Mayes PM, Garg S, Esen N. Toll-like receptor 2 modulates the proinflammatory milieu in Staphylococcus aureus-induced brain abscess. Infect Immun 2005b;73:7428-7435. [PubMed: 16239543]

Koedel U, Angele B, Rupprecht T, Wagner H, Roggenkamp A, Pfister HW, Kirschning CJ. Toll-Like Receptor 2 Participates in Mediation of Immune Response in Experimental Pneumococcal Meningitis. J Immunol 2003;170:438-444. [PubMed: 12496429]

Koedel U, Rupprecht T, Angele B, Heesemann J, Wagner H, Pfister HW, Kirschning CJ. MyD88 is required for mounting a robust host immune response to Streptococcus pneumoniae in the CNS. Brain 2004;127:1437-1445. [PubMed: 15115715]

Laflamme N, Rivest S. Toll-like receptor 4: the missing link of the cerebral innate immune response triggered by circulating gram-negative bacterial cell wall components. Faseb J 2001;15:155-163. [PubMed: 11149903]

Laflamme N, Soucy G, Rivest S. Circulating cell wall components derived from gram-negative, not grampositive, bacteria cause a profound induction of the gene-encoding Toll-like receptor 2 in the CNS. J Neurochem 2001;79:648-657. [PubMed: 11701768]

Lee S, Hong J, Choi SY, Oh SB, Park K, Kim JS, Karin M, Lee SJ. CpG oligodeoxynucleotides induce expression of proinflammatory cytokines and chemokines in astrocytes: the role of c-Jun N-terminal kinase in CpG ODN-mediated NF-kappaB activation. J Neuroimmunol 2004;153:50-63. [PubMed: 15265663]

Matsuguchi T, Musikacharoen T, Ogawa T, Yoshikai Y. Gene expressions of Toll-like receptor 2, but not Toll-like receptor 4, is induced by LPS and inflammatory cytokines in mouse macrophages. J Immunol 2000;165:5767-5772. [PubMed: 11067935]

McGeer PL, McGeer EG. Inflammation, autotoxicity and Alzheimer disease. Neurobiol Aging 2001;22:799-809. [PubMed: 11754986]

Medzhitov R. Toll-like receptors and innate immunity. Nat Rev Immunol 2001;1:135-145. [PubMed: 11905821]

Michelsen KS, Aicher A, Mohaupt M, Hartung T, Dimmeler S, Kirschning CJ, Schumann RR. The role of toll-like receptors (TLRs) in bacteria-induced maturation of murine dendritic cells (DCS). Peptidoglycan and lipoteichoic acid are inducers of DC maturation and require TLR2. J Biol Chem 2001;276:25680-25686. [PubMed: 11316801]

Nguyen MD, Julien JP, Rivest S. Innate immunity: the missing link in neuroprotection and neurodegeneration? Nat Rev Neurosci 2002;3:216-227. [PubMed: 11994753] 
Nishiguchi M, Matsumoto M, Takao T, Hoshino M, Shimonishi Y, Tsuji S, Begum NA, Takeuchi O, Akira S, Toyoshima K, Seya T. Mycoplasma fermentans lipoprotein M161 Ag-induced cell activation is mediated by Toll-like receptor 2: role of $\mathrm{N}$-terminal hydrophobic portion in its multiple functions. J Immunol 2001;166:2610-2616. [PubMed: 11160323]

Olson JK, Ludovic Croxford J, Miller SD. Innate and adaptive immune requirements for induction of autoimmune demyelinating disease by molecular mimicry. Mol Immunol 2004;40:1103-1108. [PubMed: 15036915]

Olson JK, Miller SD. Microglia initiate central nervous system innate and adaptive immune responses through multiple TLRs. J Immunol 2004;173:3916-3924. [PubMed: 15356140]

Pulendran B. Modulating vaccine responses with dendritic cells and Toll-like receptors. Immunol Rev 2004;199:227-250. [PubMed: 15233738]

Ransohoff RM, Kivisakk P, Kidd G. Three or more routes for leukocyte migration into the central nervous system. Nat Rev Immunol 2003;3:569-581. [PubMed: 12876559]

Rietze RL, Valcanis H, Brooker GF, Thomas T, Voss AK, Bartlett PF. Purification of a pluripotent neural stem cell from the adult mouse brain. Nature 2001;412:736-739. [PubMed: 11507641]

Sterka D Jr, Rati DM, Marriott I. Functional expression of NOD2, a novel pattern recognition receptor for bacterial motifs, in primary murine astrocytes. Glia 2006;53:322-330. [PubMed: 16265673]

Takeda K, Kaisho T, Akira S. Toll-like receptors. Annu Rev Immunol 2003;21:335-376. [PubMed: 12524386]

Takeuchi O, Akira S. MyD88 as a bottle neck in Toll/IL-1 signaling. Curr Top Microbiol Immunol 2002;270:155-167. [PubMed: 12467250]

Thomas PG, Carter MR, Atochina O, Da'Dara AA, Piskorska D, McGuire E, Harn DA. Maturation of dendritic cell 2 phenotype by a helminth glycan uses a Toll-like receptor 4-dependent mechanism. J Immunol 2003;171:5837-5841. [PubMed: 14634093]

Turrin NP, Rivest S. Innate immune reaction in response to seizures: implications for the neuropathology associated with epilepsy. Neurobiol Dis 2004;16:321-334. [PubMed: 15193289]

Ulevitch RJ. Therapeutics targeting the innate immune system. Nat Rev Immunol 2004;4:512-520. [PubMed: 15229470]

van der Kleij D, Latz E, Brouwers JF, Kruize YC, Schmitz M, Kurt-Jones EA, Espevik T, de Jong EC, Kapsenberg ML, Golenbock DT, Tielens AG, Yazdanbakhsh M. A novel host-parasite lipid crosstalk. Schistosomal lyso-phosphatidylserine activates toll-like receptor 2 and affects immune polarization. J Biol Chem 2002;277:48122-48129. [PubMed: 12359728]

Zanin-Zhorov A, Nussbaum G, Franitza S, Cohen IR, Lider O. T cells respond to heat shock protein 60 via TLR2: activation of adhesion and inhibition of chemokine receptors. Faseb J 2003;17:1567-1569. [PubMed: 12824285] 


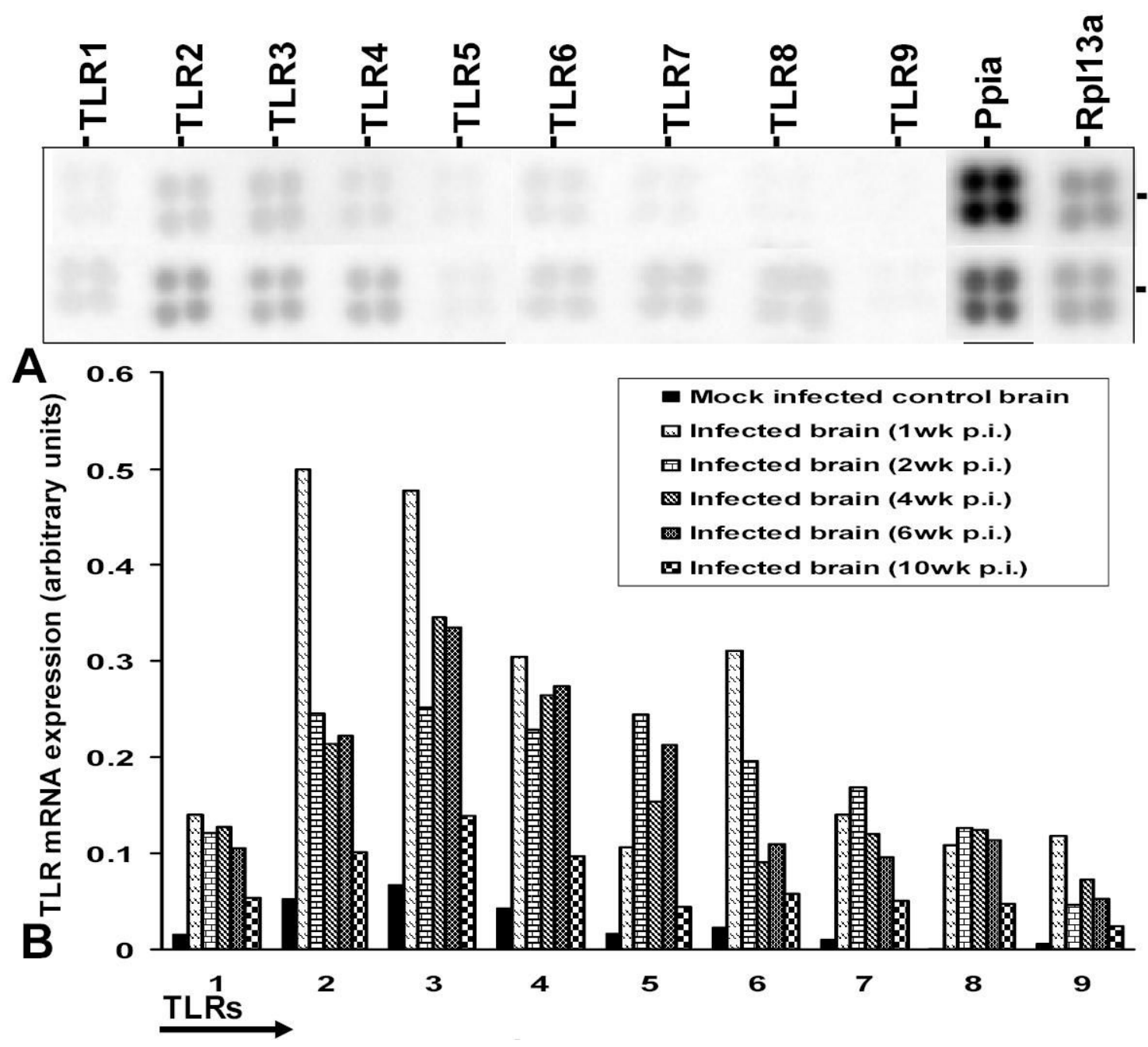

Figure 1. $M$. corti infection elevates TLR specific RNA expression levels in brain Total RNA was isolated from infected and vehicle control mouse brains at $1 \mathrm{wk}, 2 \mathrm{wk}, 4 \mathrm{wk}$, $6 \mathrm{wk}$ and 10wk p.i. using Trizol. Isolated RNA was reversed transcribed to cDNA in the presence of ${ }^{33} \mathrm{P}$-dCTP. Labeled cDNA probes were hybridized to the GEArray Q series membranes. (A) Representative NFkB pathway GEArray analysis showing expression levels of TLR1 through TLR9 and housekeeping genes pipa (cyclophilin A) and rpl13a (ribosomal protein) of RNA from brains of mock control and infected mice (boxed). (B) TLR specific RNA levels were calculated in arbitrary units and normalized to an average of the housekeeping genes cyclophilin A and rpl13a ribosomal protein. The normalized values are shown in the yaxis and represent the mean of two independent experiments. 


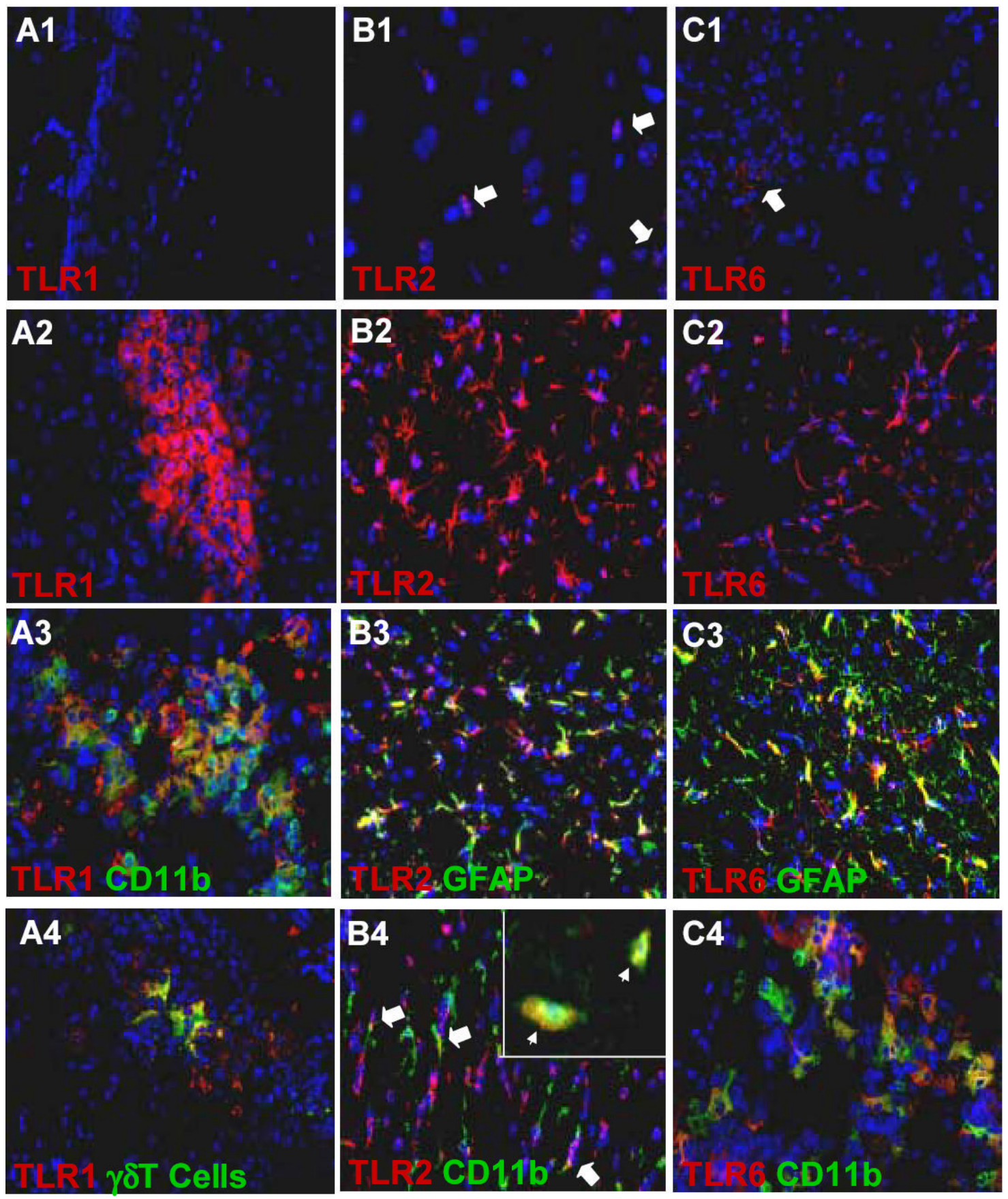

Figure 2. Distribution of TLR2 family members in brains of mock infected control and NCC mice analyzed by IF microscopy

BALB/c mice were infected intracranially with $M$. corti and sacrificed at various times p.i. After perfusion brains were removed, frozen in OCT, and cryosectioned. Sections were then analyzed for expression of TLRs and cell specific markers using fluorochrome conjugated antibodies. (A1) Mock infected control stained with TLR1 specific antibody (400X; proximity of internal leptomeninges). (A2) $M$. corti infection at $1 \mathrm{wk}$ showing TLR1 positive staining (400X; fissura longitudinalis cerebri). (A3) M. corti infection at $1 \mathrm{wk}$ showing TLR1 and CD11b+ myeloid cells, merged image (yellow/orange; 400X; meninges). (A4) M. corti infection at $1 \mathrm{wk}$ showing TLR 1 positive, $\gamma \delta \mathrm{T}$ cells, merged image (yellow/orange; 400X; 
$3^{\text {rd }}$ ventricle). (B1) Mock control mice stained with TLR2 specific antibody (arrows; 400X; cortex cerebri). (B2) M. corti infection at $1 \mathrm{wk}$ showing TLR2 positive staining (400X; caudatus putamen). (B3) $M$. corti infection at $3 \mathrm{wk}$ showing TLR2 positive astrocytes, merged image (yellow/orange; 400X; caudatus putamen). (B4) M. corti infection at $3 \mathrm{wk}$ showing TLR2 positive, microglia, merged image (arrows; 400X; corporis callosi). The insert represents a 3X magnification of a portion of the frame, depicting TLR2 colocalization with CD11b (arrow). (C1) Mock infected control stained with TLR6 antibody (arrow; 400X; vicinity of indusium griseum). (C2) M. corti infection at 1wk showing TLR6 positive staining (400X; proximity of lateral ventricle). (C3) $M$. corti infection at 3 wk showing TLR6 positive astrocytes, merged image (yellow/orange; 400X; proximity of lateral ventricle). (C4) M.corti infection at $3 \mathrm{wk}$ showing TLR6 positive, CD11b+ myeloid cells, merged image (yellow/orange; 400X; meninges). 

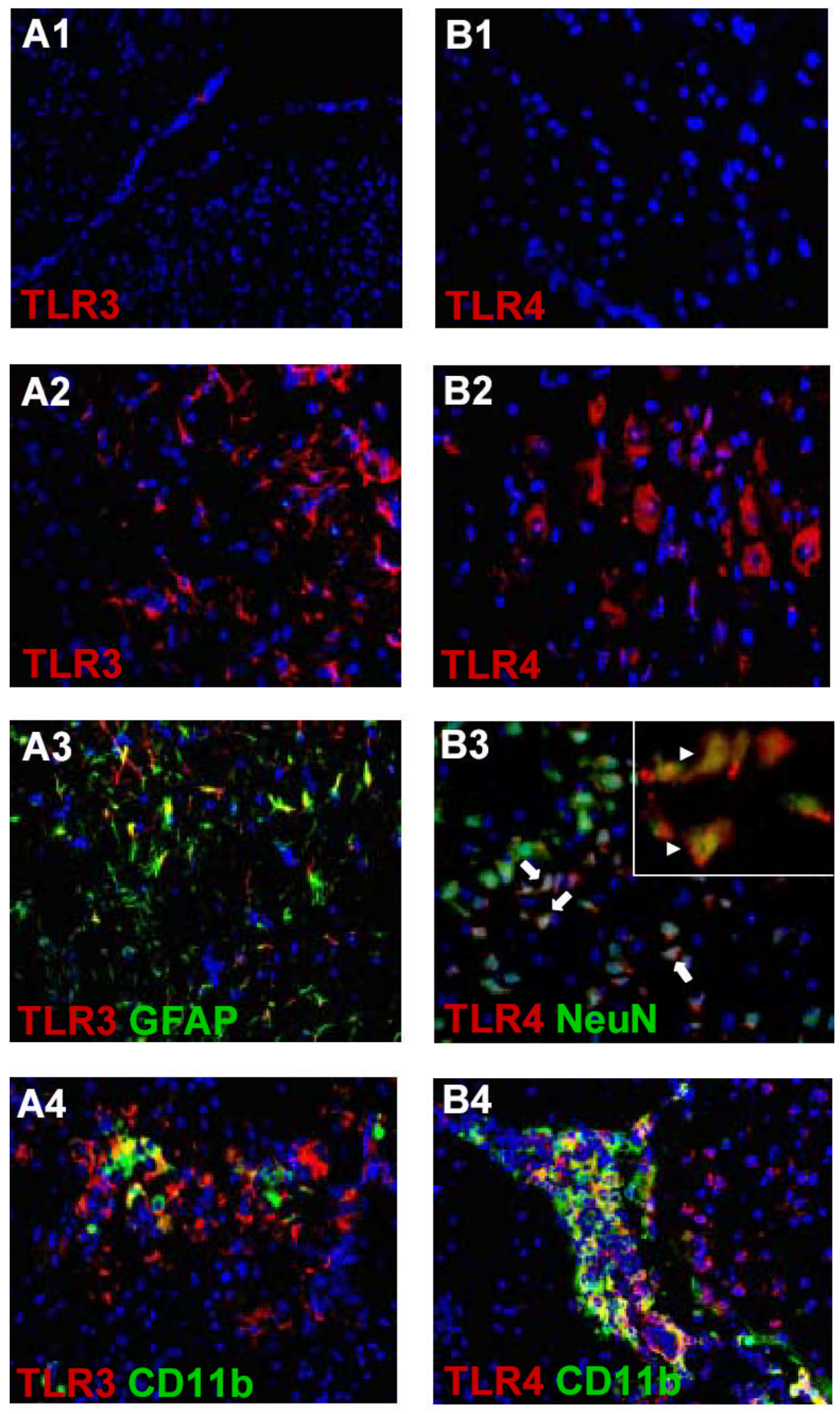

Figure 3. Distribution of TLR3 and TLR4 in brains of mock infected control and NCC mice analyzed by IF microscopy

(A1) Mock infected control stained with TLR3 specific antibody (400X; fissura longitudinalis cerebri). (A2) M. corti infection at 3wk showing TLR3 positive staining (400X; colliculi posterior). (A3) $M$. corti infection at 3 wk showing TLR3 positive astrocytes, merged image (yellow/orange; 400X; colliculi posterior). (A4) M. corti infection at $3 \mathrm{wk}$ showing TLR3 positive, CD11b+ myeloid cells, merged image (yellow/orange; 400X; meinges). (B1) Mock infected control stained with TLR4 specific antibody (400X; cortex cerebri). (B2) M. corti infection at 10wk showing TLR4 positive staining (400X; cortex cerebri). (B3) M. corti infection at $3 \mathrm{wk}$ showing TLR4 and NeuN (neuronal nuclear protein) positive staining, merged 
image (arrow; 400X; cortex cerebri). The insert represents a 3X magnification of a portion of the frame. As evident, TLR4 staining in red is in the cytosol surrounding NeuN staining in green (arrow). (B4) M. corti infection at 3 wk showing TLR4 positive, CD11b positive cells, merged image (yellow/orange; 400X; meninges). 

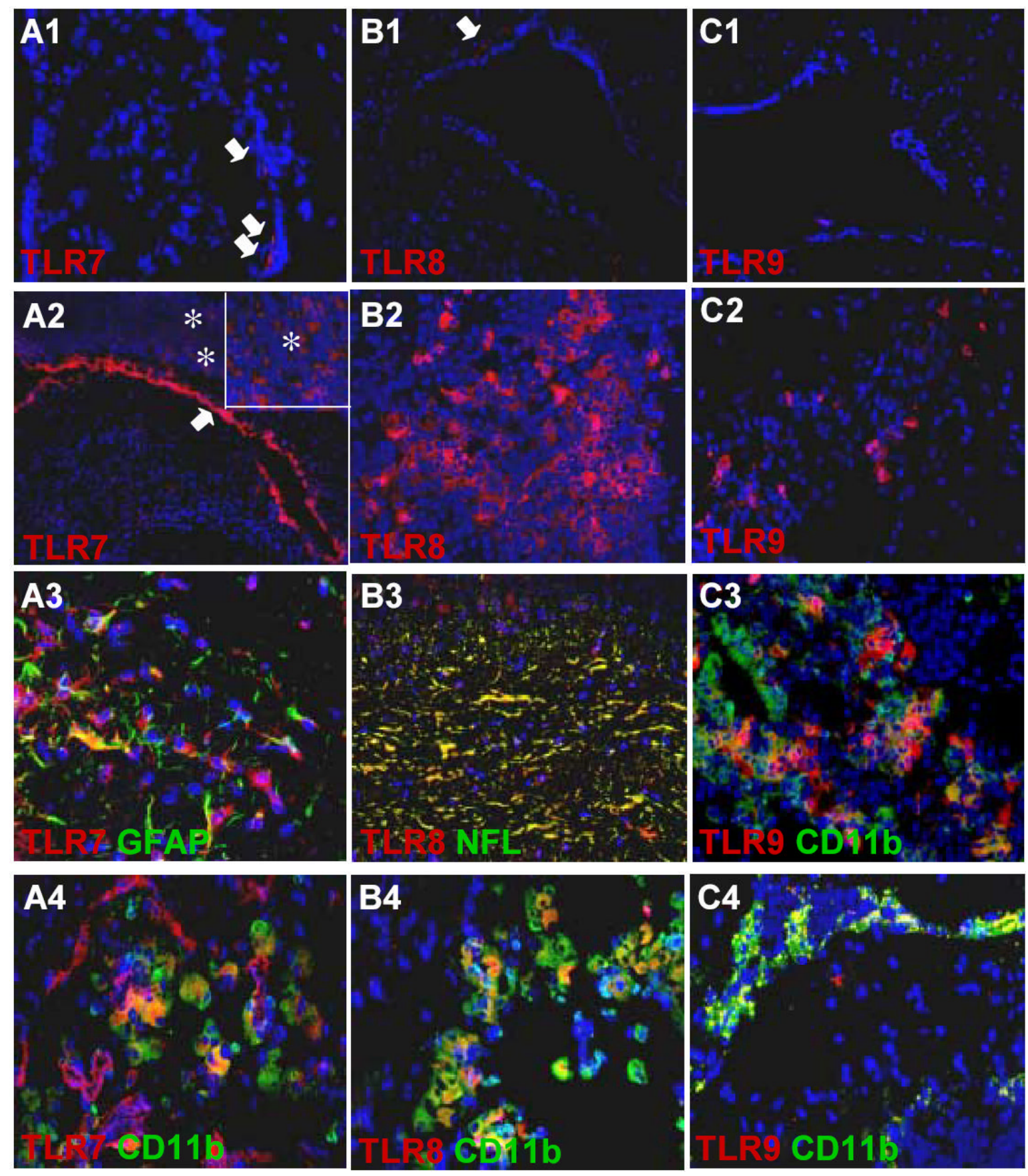

Figure 4. Distribution of TLR9 family members in brains of mock infected control and NCC mice analyzed by IF microscopy

(A1) Mock infected control stained with TLR7 specific antibody (arrow; 400X; lateral ventricle). (A2) M. corti infection at $1 \mathrm{wk}$ showing TLR7 positive ependymal cells lining the fourth ventricle (arrow; 400X; $4^{\text {th }}$ ventricle) and cerebellar granular neurons (star; 400X; cerebellum). The insert represents a $3 \mathrm{X}$ magnification of a portion of the frame confirming that granular neurons in the cerebellum are positive for TLR7 staining (star). (A3) M. corti infection at $3 \mathrm{wk}$ showing TLR7 positive astrocytes, merged image (yellow/orange; 400X; proximity of $3^{\text {rd }}$ ventricle). (A4) M. corti infection at $3 \mathrm{wk}$ showing TLR7 positive, CD11b+ myeloid cells, merged image (yellow/orange; 400X; $3^{\text {rd }}$ ventricle). (B1) Mock infected control stained with 
TLR8 antibody (arrow; 400X; meninges). (B2) M. corti infection at 3wk showing TLR8 positive infiltrating leukocytes in the ventricles (400X). (B3) M. corti infection at $3 \mathrm{wk}$ showing TLR8 and neurofilament positive staining in cerebellar white matter, merged image (yellow/ orange; 400X; cerebellum). (B4) M. corti infection at $3 \mathrm{wk}$ showing TLR8 positive, CD11b positive cells, merged image (yellow/orange; 400X; meninges). (C1) Mock infected control stained with TLR9 antibody (400X; meninges). (C2) M. corti infection at $1 \mathrm{wk}$ showing TLR9 positive infiltrating cells in the ventricle (400X). (C3) M. corti infection at $3 \mathrm{wk}$ showing TLR9 positive, CD11b positive cells in the ventricle, merged image (yellow/orange; 400X). (C4) M. corti infection at $3 \mathrm{wk}$ showing TLR9 positive $\gamma \delta \mathrm{T}$ cells, merged image (yellow/orange; $400 \mathrm{X}$; cerebellum). 


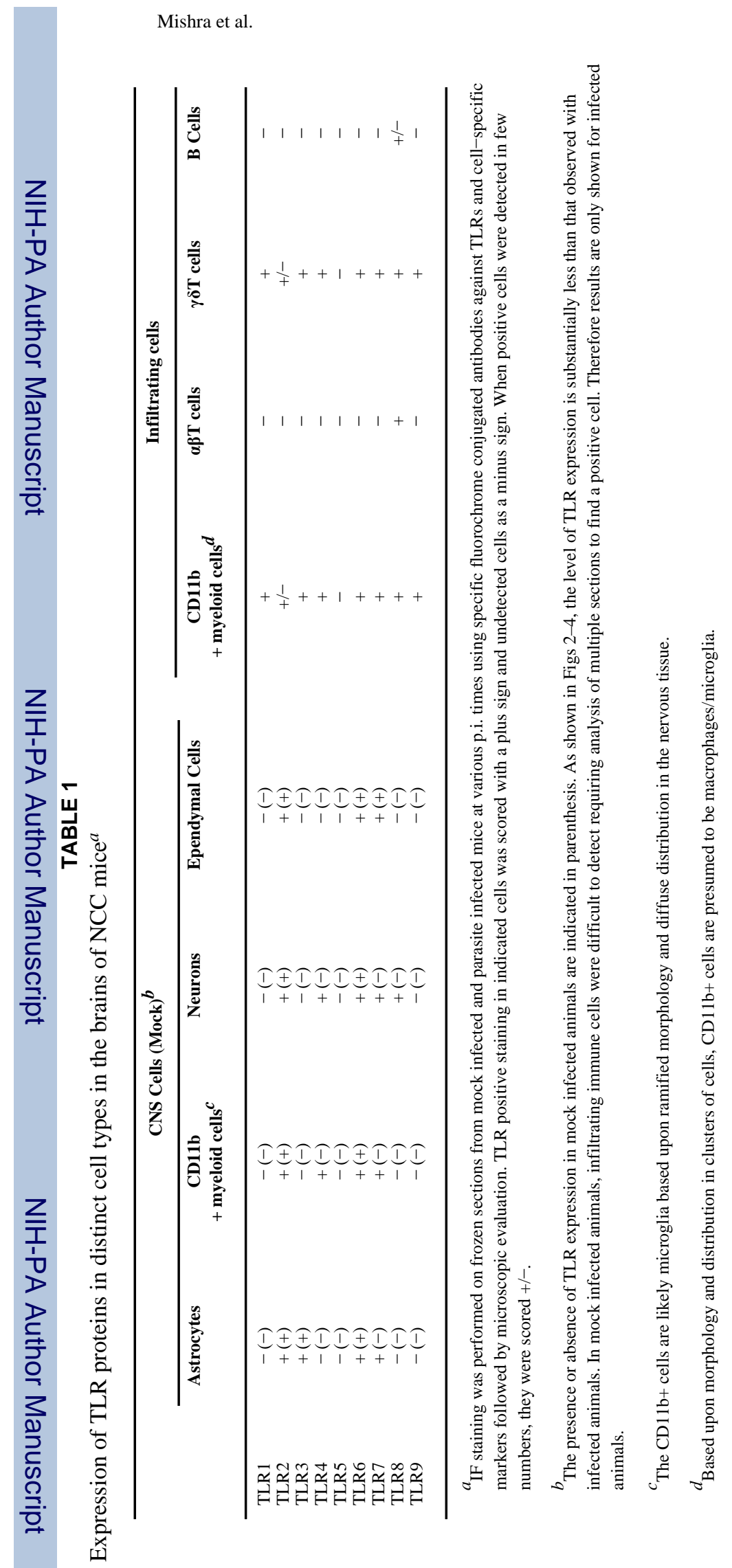

J Neuroimmunol. Author manuscript; available in PMC 2007 December 1. 